\title{
Calculation of the Critical Current Reduction in a Brittle Round Multifilamentary Wire due to External Forces
}

\author{
Bennie ten Haken, Arno Godeke and Herman H. J. ten Kate \\ Applied Superconductivity Centre, University of Twente, \\ P.O. Box 217, 7500 AE Enschede, The Netherlands
}

\begin{abstract}
A stmple model is presented that can describe the electromechanical state of a multifilamentary wire. An elastic cylinder model is used to derive the strain state analytically. Arial and transverse forces cause a position dependent critical current density in the wire. The Integral critical current of such a wire is calculated. The effect of two different parameters, the hydrostatic and the deviatoric strain, on the critical current is compared. The critical current reduction of a model wire due to various erternal loads in a $\mathrm{Nb}_{3} \mathrm{Sn}$ wire is analysed. Finally the position of the superconducting filaments is considered.
\end{abstract}

\section{INTRODUCTION}

High field superconductors are exposed to large pressures caused by thermal contraction and the Lorentz-forces inside high performance magnets. The critical current in many of these conductors is affected by the deformation of the superconducting area. The dependence of the critical current density on the strain is well investigated for $\mathrm{Nb}_{3} \mathrm{Sn}$ and other A15 conductors. Many ceramic superconductors, such as $\mathrm{Bi}-2212$, also show a strain dependent critical current.

The critical current of a superconductor in its operating environment is calculated in two steps. First the internal strain state is analysed to predict the local critical current density. Then the integral critical current of the wire is calculated with Maxwell's equations using special constitutive equations for the super- and normal-conducting areas. The intrinsic critical current density versus strain relation, necessary to solve this model, may change for each material.

\section{THE MODEL WIRE}

The first step is to set-up a mechanical and electrical model, to approximate the critical current $\left(I_{c}\right)$ of a real superconducting wire in its operating environment. Here we focus on analytical solutions. Extensive numerical computations, who could simulate the actual geometry more accurate, are left for a later exercise.

\section{A. Definitions}

We consider a round wire with a normalised radius $(R=1)$. The superconductor is concentrated in a cylinder with a certain inner and outer radius $\left(0<R_{1}<R_{2}<1\right)$. The area outside the superconducting (SC) area is normal conducting. The material properties of these regions are listed

Manuscript received September 20, 1993 in Table 1 . Inside the wire the cylindrical co-ordinate system is used $(r, \varphi, z)$, but occasionally the Cartesian system is preferred with $x=r \cos (\varphi)$ and $y=r \sin (\varphi)$. The wire axis coincides with the $z$-axis.

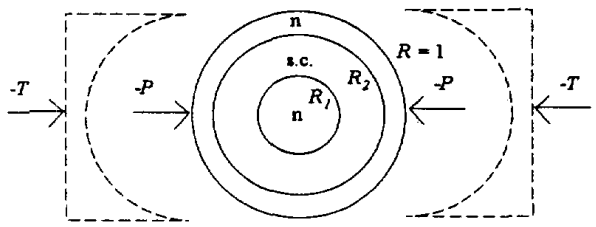

Fig. 1: The cross sectional view of the model wire model. Also indicating both the transverse external loads $P$ (line) and $T$ (uniform)

Mechanical properties: Only the isotropic elastic case is considered here and for simplicity the elastic properties are equal and constant in both the areas. A difference in the thermal contraction between the superconductor and the matrix material is taken into account.

Electrical properties: The electrical properties of the SCarea imply a strong anisotropic behaviour. A superconducting and a resistive current flows parallel to the twisted filaments. The voltage-current characteristic of the superconductor is described later. In the direction transverse to the filaments flow only resistive currents.

Mechanical loads: The mechanical state is simplified to a constant thermal contraction, plus a combination of external forces. In the $z$-direction an axial strain is applied. In the $x$ direction the influences of a concentrated force $P$ and a uniform force $T$ are considered.

TABLE 1:

DEFINTTION OF THE MATERIAL PROPERTIES

\begin{tabular}{lcc}
\hline Parameter & S.C.- area & Normal \\
\hline Young's modules & $E_{\text {mod }}$ & $E_{\text {mod }}$ \\
Poison ratio & $v$ & $v$ \\
Integral thermal contraction & $\delta_{s c}$ & $\delta_{n}$ \\
Critical current density & $J_{c}$ & - \\
Twist pitch angle & $\beta$ & - \\
Resistivity parallel to filaments & $\rho_{p f}$ & $\infty$ \\
Resistivity transverse to filaments & $\rho_{t f}$ & $\infty$ \\
\hline
\end{tabular}




\section{A CYLINDRICAL ELASTIC BODY}

The principle procedure for calculating the strain in an elastic cylinder is found in any good textbook on continuum mechanics. The case were a rotational symmetry is maintained is presented first. Then the influence of a transverse force is calculated. The symbols $\varepsilon$ and $\sigma$, with an index for the co-ordinate denote the strain and stress respectively.

\section{A. Rotational symmetric solutions.}

There exists a general rotational symmetric solution for the stress in the three elastic cylinders of this model. The following restrictions at the interfaces yield the solution: $\varepsilon_{z}$, $\varepsilon_{\phi}$ and $\sigma_{r}$ are continues at $r=R_{1}$ and $R_{2}$, the strain in the centre is finite and $\sigma_{r}$ is zero at the wire surface. The strain inside the $\mathrm{SC}$-area is presented in terms of strain as a function of the axial strain $\left(\varepsilon_{a}\right)$ applied after cooling down:

$$
\begin{aligned}
& \varepsilon_{r}=\frac{\delta_{s c}-\delta_{n}}{2(1-v)}\left[A_{n}(1-3 v)-\left(R_{l} / r\right)^{2}(1+v)\right]-v \varepsilon_{a}, \\
& \varepsilon_{\phi}=\frac{\delta_{s c}-\delta_{n}}{2(1-v)}\left[A_{n}(1-3 v)+\left(R_{l} / r\right)^{2}(1+v)\right]-v \varepsilon_{a}, \\
& \varepsilon_{z}=\left(\delta_{s c}-\delta_{n}\right) A_{n}+\varepsilon_{a}, \\
& A_{n}=1+R_{l}^{2}-R_{2}^{2} .
\end{aligned}
$$

In this case the shear strains are zero, so the hydrostatic and the deviatoric strains $\left(\varepsilon_{\text {hyd }}, \varepsilon_{\text {dev }}\right)$ can be written as:

$$
\begin{aligned}
& \varepsilon_{\text {hyd }}=\left(\varepsilon_{r}+\varepsilon_{\phi}+\varepsilon_{z}\right) / 3, \\
& \varepsilon_{d e v}=\frac{2}{3} \sqrt{\left(\varepsilon_{r}-\varepsilon_{\phi}\right)^{2}+\left(\varepsilon_{\phi}-\varepsilon_{z}\right)^{2}+\left(\varepsilon_{z}-\varepsilon_{r}\right)^{2}} .
\end{aligned}
$$

The hydrostatic strain is constant in the SC-area. It is zero at an applied axial strain $\left(\varepsilon_{0^{\prime}}\right)$ of:

$$
\varepsilon_{o^{\prime}}=2 A_{n} \frac{\delta_{n}-\delta_{s c}}{1-v}
$$

The deviatoric strain is a function of the radius with a minimum for a certain applied strain. The strain where this minimum appears $\left(\varepsilon_{0^{\prime \prime}}\right)$ is independent of the radius:

$$
\varepsilon_{o^{\prime \prime}}=\frac{A_{n}}{2} \frac{\delta_{n}-\delta_{s c}}{1-v}=\frac{1}{4} \varepsilon_{o^{\prime}}
$$

\section{B. Transverse forces}

Uniform pressure: First a uniaxial transverse pressure $(T)$ in the $x$-direction is considered, as if the cylinder is enclosed in an infinite body with similar elastic properties. The axial strain is taken as zero $\left(\varepsilon_{z}=0\right)$ and the $y$-direction is stress free $\left(\sigma_{y}=0\right)$. These conditions yield the following solution in the Cartesian co-ordinate system:

$$
\varepsilon_{x}=\frac{T}{E}\left(1-v^{2}\right) \cos ^{2}(\phi), \varepsilon_{y}=\frac{T}{E}\left(-v-v^{2}\right) \sin ^{2}(\phi) .
$$

Line force: In a second case a transverse line pressure $(P)$ is acting on the surface $(r=1)$, in the $x$-direction at $\varphi=0$ and $\pi$, by which the rest of the surface is force free. There exists an analytical solution for the stress in this case [1], which is combined with the condition $\varepsilon_{z}=0$ :

$$
\begin{aligned}
& \sigma_{r}=f\left(r^{2}-1\right)\left(2 \cos (2 \phi)-\left(r^{4}+2 r^{2}-1\right)\right), \\
& \sigma_{\phi}=f\left(2\left(2 r^{4}+r^{2}+1\right) \cos (2 \phi)-\left(r^{6}+r^{4}+5 r^{2}+1\right)\right), \\
& \sigma_{r \phi}=-f\left(r^{2}-1\right)\left(r^{4}-1\right) \sin (2 \phi), \\
& f=\frac{P}{\pi}\left(r^{2}-1\right)\left(2 r^{2} \cos (2 \phi)-\left(r^{4}+1\right)\right)^{-2} .
\end{aligned}
$$

Note that for a compressive $x$-stress $P$ and $T$ are negative.

\section{Calculation of the InTegral Critical CurRent}

The critical current of the wire considered is a function of the local critical current density $\left(J_{c}=J_{c}(r, \varphi)\right)$, the voltage current characteristic of the superconductor and the resistivity of the matrix material. The currents in the normal conducting core and outer shell are disregarded, but the normal currents in the matrix between the filaments are taken into account. A logarithmic behaviour describes the superconducting current density in the filament region, as a function of the electrical field $\left(E_{p p}\right)$ parallel to filaments:

$$
J_{s}(r, \phi)=J_{c}(r, \phi)+J_{1} \ln \left(E_{p f}(r, \phi) / E_{c}\right) \text {. }
$$

A good approximation of the currents in the filaments can be found by disregarding the currents in the radial direction $\left(J_{r}=0\right)$. Then the solution is simplified to the current distribution in a cylinder with a certain radius (r) and twisted filaments. In the next section an alternative is sketched to the approach of Polak et al.[2]. First we consider the current densities parallel $\left(J_{p j}\right)$ and transverse $\left(J_{t j}\right)$ to the filaments:

$$
J_{p f}=E_{p f} / \rho_{p f}+J_{s} \text { and } J_{t f}=E_{t f} / \rho_{t f},
$$

where $E_{t f}$ is the electrical field transverse to the filaments. Using $z$-invariance one can derive [2]:

$$
d J_{\phi}(\phi) / d \phi=0 \text { and } \int_{0}^{2 \pi} E_{\phi}(\phi) d \phi=0
$$

A rotation of the equations for the current to the cylindrical co-ordinate system, yields the following equation for the electrical field parallel to the filaments: 


$$
E_{\phi}=E_{z} \cos (\beta)+\rho_{e f f}\left(\int_{0}^{2 \pi} J_{s}(\phi) d \phi-J_{s}(\phi)\right),
$$

$$
\text { with: } \frac{1}{\rho_{e f f}}=\frac{1}{\rho_{p f}}+\frac{1}{\rho_{t f} \tan ^{2}(\beta)} \text {. }
$$

Combining the equations (16) and (17), an expression that determines the current density profile is obtained:

$$
E_{z} \cos (\beta)=e^{\frac{J_{s}(\phi)-J_{c}(\phi)}{J_{1}}}-\rho_{e f f}\left(\int_{0}^{2 \pi} J_{s}(\phi) d \phi-J_{s}(\phi)\right) .
$$

This integral equation is solved numerically with a Newton iteration over a discrete number of points along the angular co-ordinate. An integration of the total current in the SCarea finally yields the current of the wire.

\section{Practical applications}

An important application to consider is a multifilamentary $\mathrm{Nb}_{3} \mathrm{Sn}$ wire. For this study a model wire with the following dimensions: $R_{1}=0.25, R_{2}=0.75$ and a twist pitch $\beta=0.1 \pi$ is considered. The integral thermal contraction difference is: $\left(\delta_{s c}-\delta_{n}\right)=-0.4 \%$. The following properties are normalised: $E_{c}=1, J_{c}(r, \varphi) \approx 1, J_{1}=10^{-2}$, $\rho_{\text {eff }}=10^{-3}, E_{\text {mod }}=1$. The poisson ratio is taken as $v=0.3$.

\section{A. $J_{c}(\varepsilon)$ dependence.}

Even for a well investigated material as $\mathrm{Nb}_{3} \mathrm{Sn}$ the critical current density as a function of the strain tensor is not accurately known. In the case of a tensile axial strain Ekins "scaling law" for the upper critical field $B_{c 2}$ is a good fit [3]. However for strong compressive axial strains a linear reduction of the $B_{c 2}$ is found. A similar behaviour is found if a transverse stress is applied to $\mathrm{a} \mathrm{Nb}_{3} \mathrm{Sn}$ layer [4].

For the generalisation of the $B_{c 2}$ dependence towards the six component strain tensor the first two invariants are proposed; the hydrostatic and deviatoric strain. Welch [5] concluded for $\mathrm{Nb}_{3} \mathrm{Sn}$ that the influence of the deviatoric strain is dominant. However experimental results obtained on a $\mathrm{Nb}_{3} \mathrm{Sn}$ layer, deformed by a combination of external loads, do not confirm this conclusion [4]. Therefore both the strain invariants are considered here.

Because the emphasis is on compressive strains, the linear approximation of the $B_{c 2}$ versus strain relation is applied:

$$
\begin{aligned}
& B_{c 2}\left(\varepsilon_{\text {hyd }}\right)=B_{o h}+C_{h} \varepsilon_{h y d}, \\
& B_{c 2}\left(\varepsilon_{d e v}\right)=B_{o d}+C_{d} \varepsilon_{d e v} .
\end{aligned}
$$

The critical current density is determined by the upper critical field with the Kramer relation for $J_{c}(B)$ :

$$
J_{c}(B)=\frac{C_{p}}{B}\left(B / B_{c 2}\right)^{-1 / 2}\left(1-B / B_{c 2}\right)^{2} \text {. }
$$

All the constants will be defined in the next paragraph.

\section{B. Axial strain case: Hydrostatic and Deviatoric strain}

In order to compare the different strain functions the properties of the standard wire after cooling down are defined as: $I_{c}=1, B_{c 2}=22 \mathrm{~T}$. The right a slope of the $B_{c 2}$ versus $\varepsilon_{a}$ relation of $8 \mathrm{~T} / \%$, at $\varepsilon_{a}=0$, is obtained with:

$$
C_{h}=800\left(\frac{3}{1-2 v}\right) \text { and } C_{d}=-800 \text {. }
$$

The other constants are defined by the critical current at the point where $\varepsilon_{a}=0$. In the deviatoric approach the constant $B_{o d}$ is defined by the mean value of the $B_{c 2}$ in the superconducting area.

The resulting critical current versus strain relation is plotted in Fig. 2. The value and derivative of the current at zero strain are of course equal for both $B_{c 2}$ approaches. For positive strain the hydrostatic approach shows a continuously increasing critical current. The deviatoric approach shows a maximum in the current at the position where the minimum in this strain occurs. Remind that this is before the point where the axial strain is zero. For a negative applied strain the differences are rather small.

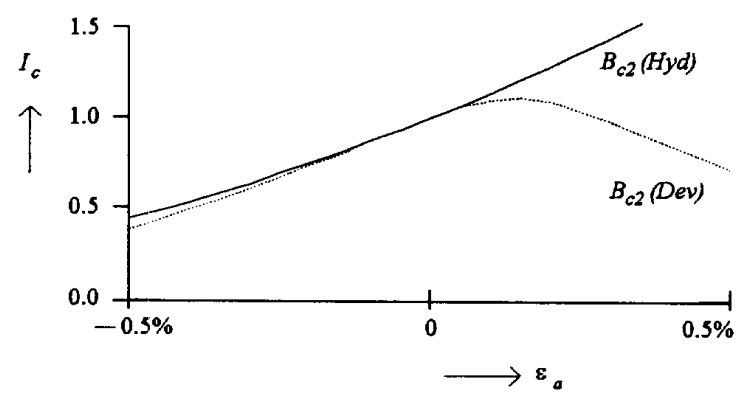

Figure 2: Critical current versus the applied axial strain for the two $B_{c 2}$ versus strain relations.

\section{Transverse pressure}

The applied axial strain is taken as zero in this consideration. First the influence of the force distribution is investigated. The influence of the position of the SC-area in the wire section on the $I_{c}$ after thermal compression is considered at the end.

Force distribution. A uniform transverse force $T$, (with $P=0$ ), is compared with a line force $P$ (with $T=0$ ) and a 
combination of both $T=P$ is considered. In the first uniform case the hydrostatic strain is constant in the SCarea, otherwise the hydrostatic strain is position dependent. The deviatoric strain is always position dependent. The overall critical currents are plotted in Fig. 3.

If the deviatoric approach is used the combined load $(T=P)$ shows the smallest current reduction. In case of a concentrated load strain concentrations at $R_{2}$ and in case of a uniform stress strain concentrations at $R_{l}$, both in combination with the thermal stress, cause a stronger current reduction. Because of the linear properties of the hydrostatic strain the influence of an axial strain and a transverse pressure results in a similar $I_{c}$ reduction. The differences due the various pressure profiles are very small in this example $(<1 \%)$.

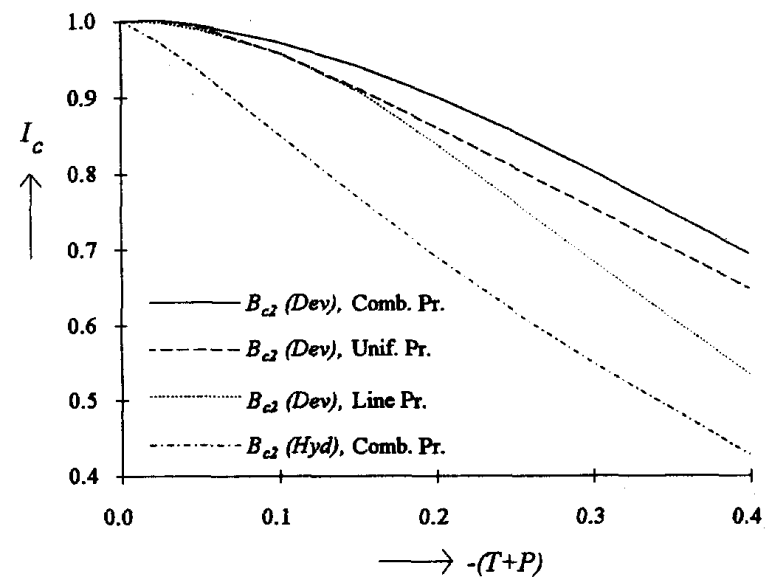

Figure 3: The influence of various transverse pressures on the critical current.

Changing $R_{1}$ and $R_{2}$ : The influence of the position of the SC-area inside the wire is investigated while remaining a constant SC/Normal ratio. Thus the value of $R_{2}$ may be changed from $\sqrt{2}$ to 1 . If the constants describing the Deviatoric approach are not adjusted, then the $I_{c}$ after thermal compression is influenced by this change. The change in $I_{c}$ is caused by the term $R_{l} / r$ in equations (1) and (2). This term implies that the maximum in the deviatoric strain appears at $R_{l}$, but by reducing the radius the area with large deviatoric strains is thus reduced. The $I_{c}$ after cooling down changes approximately $30 \%$ in this example (see Fig. 4).

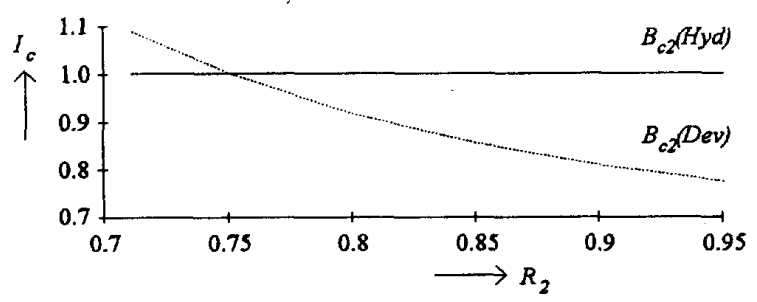

Figure 4: The $I_{c}$ due to the thermal pre compression as a function of the $R_{2}$.
In Fig 5 the influence of a combined constant pressure $(P=T=-0.1)$ is plotted. The $I_{c}$ after cooling down is normalised at 1 . The current reduction is stronger if the SCarea is placed at the outside of the conductor, but the total change is relatively small.

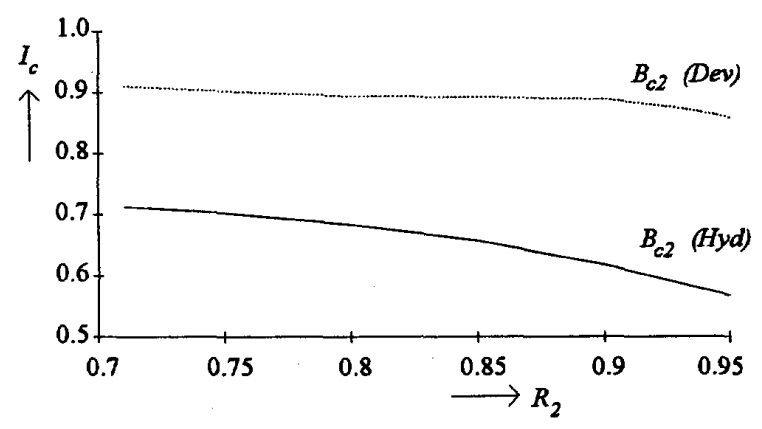

Figure 5: The $J_{c}$ reduction at a transverse pressure $(P=T=-0.1)$.

\section{CONCLUSIONS}

1 - A simple model is developed that can predict the integral critical current of a multifilamentary wire, influenced by a combination of external mechanical loads.

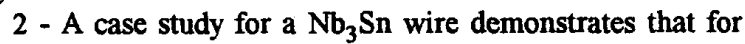
compressive axial strains, the difference between the hydrostatic and the deviatoric approach is very small.

3 - When axial tensile strains are applied, there appears a minimum in the deviatoric strain before the axial thermal strain is cancelled. In the deviatoric approach this minimum produces a maximum in the critical current.

4 - In the case of a transverse compressive strain the $I_{c}$ reductions found for small strains is essentially different, the hydrostatic approach shows a stronger decrease than the deviatoric approach.

5 - A change in the position of the SC-area inside the wire causes a change of the deviatoric strain induced by thermal contraction. In the example wire the $I_{c}$ calculated with the deviatoric approach reduces with $30 \%$, if the $\mathrm{SC}$ is moved towards the centre.

\section{REFERENCES}

[1] N.I. Muskhelishvili, "Some basic problems of mathematical theory of elasticity", Noordhof Groningen, the Netherlands, 1963.

[2] M. Polak, R. G. Mints and Majoros, "Influence of filament spirality on the current voltage characteristics of twisted multifilamentary NbTi superconductors", Cryogenics, Vol. 29, pp 963-970, 1989.

[3] J.W. Ekin, "Four-dimensional J-B-T-E critical current surface for superconductors", J. Appl. Phys, vol 54, pp 303-306, 1983.

[4] B. ten Haken A Godeke and H.H.J. ten Kate, "The influence of various strain components on the critical parameters of layer shaped $\mathrm{Nb} 3 \mathrm{Sn}^{\prime \prime}$, Proc. ICMC-1993, in press.

[5] D.O. Welch, "Alternation of the superconducting properties of A15 compounds and elementary composite superconductors by nonhydrostatic elastic strain", Adv. in Cry. Eng. Vol. 26, pp 48-65, 1980. 